\title{
EL DIAGNÓSTICO EN EDUCACIÓN COMO PRINCIPIO DE IDENTIFICACIÓN EN EL ÁMBITO DE LA SUPERDOTACIÓN INTELECTUAL
}

\author{
ASSESSMENT IN EDUCATION AS PRINCIPLE OF IDENTIFICATION \\ IN THE AREA OF INTELLECTUAL GIFTEDNESS
}

\author{
Ana $M^{a}$ Peña del Agua* \\ Universidad de Oviedo
}

\begin{abstract}
RESUMEN
Educar en diversidad es un requisito importante que contempla nuestro sistema educativo, ya que se han de tener en cuenta las características propias de todos los estudiantes para llevar a cabo una educación con mayor eficacia y calidad. Las premisas que apoyan la atención educativa a la superdotación derivan del reconocimiento y aceptación de las diferencias individuales. El superdotado es diferente en su potencial cognitivo, aprende más, con mayor rapidez, profundidad y amplitud. En este artículo queremos abordar la importancia del diagnóstico como procedimiento de identificación desde las características individuales de los sujetos con alta capacidad. En él también destacamos la importancia de los instrumentos de medida y fuentes de información más relevantes relacionadas con la evaluación diagnóstica. Finalizamos con algunas consideraciones metodológicas sobre el uso del estudio de casos como estrategia de investigación para integrar la información, llegar a la búsqueda de significado y a la toma de decisiones.
\end{abstract}

Palabras claves: Diversidad, superdotación intelectual, diagnóstico, instrumentos de medida, fuentes de información, estudio de casos.

\begin{abstract}
Educating in diversity is an important requirement of our educational system, as all students' own characteristics must be taken into account in order to fulfil the education with greater efficiency and quality. The premises that support the educational attention to giftedness descend from the recognition and acceptance of individual differences. The gifted one is different as regards the cognitive potential, learning more, faster, in more depth and width. In this article, we aim to focus on the importance of the assessment as the proper procedure of identification of the individual characteristics of people

\footnotetext{
* Ana María Peña del Agua es Profesora Titular de Diagnóstico y Evaluación en Educación. Departamento de Ciencias de la Educación de la Universidad de Oviedo. Área MIDE. Líneas de investigación: diagnóstico e intervención en necesidades educativas específicas. delagua@uniovi.es
} 
who show high abilities. We also want to point out the importance of the instruments of measure and the most relevant sources of information related to the diagnostic evaluation. We will finish with some methodological considerations about the usefulness of the survey in different cases as strategy of investigation in order to gather the information, reach the search of meaning and make decisions.

Key words: Diversity, intellectual giftedness, assessment, measure instruments, sources of information, study the cases

\section{Introducción}

La superdotación es un concepto que sirve para denominar un alto nivel de inteligencia e implica un desarrollo avanzado y acelerado de las funciones del cerebro (Clark, 2000). Dicho desarrollo se manifiesta mediante altos niveles de habilidades o capacidades en cuanto a cognición, metacognición, aptitud académica, creatividad e innovación, habilidades personales e interpersonales, liderazgo, artes visuales y artísticas. Superdotado es, pues, aquel sujeto que demuestra tener aptitudes para ejecutar dichas áreas a altos niveles; y este tipo de sujetos precisa un entorno de aprendizaje adecuado tanto en el ámbito escolar como en el familiar, para que sus habilidades se desarrollen al máximo.

El concepto actual de superdotación supone un cambio respecto de las creencias anteriores sobre la inteligencia. Hasta hace algunos años se pensaba que la inteligencia no cambiaba a lo largo de la vida y que disminuía con la madurez. A partir de un gran número de estudios sobre su desarrollo sabemos que el cerebro no sólo transforma la inteligencia de forma interactiva, sino que también responde a la intensidad de una adecuada estimulación o a la inhibición por falta de oportunidades, y que sigue siendo dinámica a lo largo del ciclo vital (Diamod, 1988; Kandel y Schwartz, 1991). Los profesionales que trabajan con niños con alta capacidad deben entender la importancia de la interacción entre lo genético y el entorno, considerando el desarrollo de la inteligencia como proceso dinámico en el que cobra gran importancia el tipo de planificación educativa. Este planteamiento lleva a considerar la necesidad de identificar a través de un diagnóstico ajustado las diversas formas de intervención que pueden contribuir a un mayor desarrollo de las capacidades, para lo que se hace necesario llevar a cabo una atención a la diversidad individual apropiada a las características de estos estudiantes.

\section{Indicadores que facilitan el reconocimiento del alumnado con altas capacidades}

Las capacidades superiores son un concepto amplio y complejo que abarca una gran variedad de características. Los diversos autores especializados en superdotación han propuesto distintas clasificaciones de las principales características que pueden ser observadas en los alumnos superdotados (Bloom, 1985; García Yagüe y col. 1986; Coriat, 1990; Clark, 1992; Webb, 1993). Tales rasgos son generalizaciones obtenidas a partir de poblaciones generales de alumnos superdotados, por lo que también se puede dar superdotación sin ellos (Howell y otros, 1997).

Whitmore (1988) ha señalado la existencia de características observables en niños con altas habilidades que incluso en ausencia de un elevado rendimiento pueden indicar super- 
dotación. Según el citado autor tales características se derivan de las principales categorías de la conducta inteligente. A continuación enumeramos algunos de los indicadores propuestos por Whitmore, que con frecuencia se observan en estos alumnos. Tales indicadores han sido agrupados en primarios y secundarios, y facilitan el reconocimiento de los estudiantes con altas capacidades.

\section{Indicadores primarios}

Estos indicadores se refieren principalmente a todo lo relacionado con el intelecto, altos niveles de capacidades cognitivas y metacognitivas. Seguidamente se reflejan algunos de los mismos:

- Aprendizaje rápido y fácil cuando está interesado.

- Capacidad cognitiva excepcional para aprender, retener y utilizar los conocimientos.

- Destreza superior para resolver problemas. Utiliza el conocimiento adquirido y las destrezas de razonamiento superior para resolver problemas complejos, teóricos y prácticos.

- Incorporación al lenguaje oral de un vocabulario avanzado, que utiliza de un modo adecuado, y una estructura lingüística compleja.

- Comprensión excepcional de ideas complejas y abstractas (elaborando ideas a un nivel no esperado).

- Nivel elevado de indagación en los temas que despiertan su interés y mantienen su atención.

- Calidad excepcional del pensamiento, como se revela a través del lenguaje y de la capacidad para resolver problemas.

- Manipulación notable de símbolos e ideas abstractas, incluyendo la percepción y manejo de las relaciones entre ideas, sucesos y personas.

- Formulación de principios y generalizaciones gracias a la transferencia de aprendizaje a través de situaciones o acontecimientos.

- Reflexión y razonamiento para lograr intuiciones y generar soluciones.

\section{Indicadores secundarios}

Los indicadores secundarios están orientados a aspectos como la creatividad e innovación, los intereses personales, la motivación, las habilidades personales e interpersonales. A continuación señalamos, entre otros, los siguientes:

- Comportamiento sumamente creativo en la producción de ideas, objetos y soluciones; puede ser notablemente creativo e inventivo (originalidad).

- Extensa gama de intereses; básicamente son muy curiosos.

- Interés profundo y a veces apasionado por una o diversas áreas de investigación intelectual.

- Intenso deseo de conocer y entender, dominar destrezas y problemas de interés. 
- Manifestación de iniciativa para seguir proyectos ajenos. Puede elaborar hobbies según su propia elección. Manifiesta inventiva y una capacidad excepcional para el aprendizaje autodirigido, aunque posiblemente sólo en actividades extraescolares.

- Complacencia en la auto-expresión, especialmente a través de la discusión, pero con frecuencia también por medio del arte.

- Independencia en el pensamiento, una tendencia hacia la no-conformidad.

- Exigencia de una razón o explicación de los requisitos, límites y sucesos no deseados.

- Tendencia al perfeccionismo, es intensamente autocrítico y aspira a niveles elevados de rendimiento; desea sobresalir y producir.

- Manifiesta una gran sensibilidad y consciencia con respecto a sí mismo, a los demás, los problemas del mundo y las cuestiones morales; puede resultar intolerante con la debilidad humana.

Partiendo del supuesto de que los sujetos con altas habilidades disponen de características específicas, entendemos que es necesaria la identificación a través de un diagnóstico para dar una respuesta que garantice la mejor adaptación del proceso enseñanza y aprendizaje a los intereses, motivaciones, necesidades personales y sociales de este colectivo.

\section{Identificación y diagnóstico del superdotado}

La identificación de los niños superdotados ha sido y es un tema muy controvertido, ya que al no existir un modelo perfecto y/o único, puede suceder que niños muy capaces no sean identificados: es el caso de los niños denominados por Hany (1993) "falsos negativos", por lo mismo puede suceder lo contrario, a saber, que niños que no son superdotados puedan ser identificados como tal, y a ellos se les califica de "falsos positivos". La identificación y el diagnóstico son cuestiones complejas fundamentalmente por dos razones: la primera depende de la definición que se adopte previamente, es decir, qué se entiende por superdotación intelectual, mientras que la segunda hace referencia a las técnicas e instrumentos de evaluación que se utilicen, y en particular a la fiabilidad y validez de los mismos. Las dimensiones que se desprendan del concepto de superdotación adoptado determinarán las variables, el contexto de aplicación, la metodología, las técnicas que se utilicen y la propia situación de medida.

Para Jiménez Fernández (2000) la identificación significa un juicio global sobre la potencial capacidad, mientras que el diagnóstico trata de especificar los recursos singulares de cada uno de los alumnos ya identificados como de baja, media o alta capacidad. Según esto, el diagnóstico es un proceso más amplio y preciso que la identificación, comprende a ésta y forma parte del proceso educativo. En cualquier caso, el diagnóstico aporta información útil sobre aspectos de desarrollo, maduración, estilos de aprendizaje de los alumnos, considerados individualmente o en grupo, por lo que creemos, de acuerdo con Treffinger y Feldhusen (1996), que debe entenderse como un proceso continuo, no como un proceso único que afirma de una vez y para siempre si un niño es superdotado o no. Así pues, el diagnóstico proporcionará información relevante para la toma de decisiones sobre una adecuada intervención y su correspondiente evaluación. 


\section{Carácter individual de la superdotación}

La superdotación es un fenómeno que puede ser observado en los sujetos, por lo que se trata de una característica individual. Este supuesto conduce al intento de medir aspectos del sujeto, y a partir de ellos realizar afirmaciones concretas sobre su capacidad. La atención a las diferencias específicas surge una vez que éstas han sido contrastadas y verificadas a través de un proceso de indagación basado en una metodología adecuada. Por otra parte, la planificación y el diseño de programas educativos se lleva a cabo teniendo en cuenta estas características individuales. La identificación del superdotado y de los talentosos, desde un punto de vista general (Berché, 1999) precisa de dos características importantes: la primera es que sólo puede validarse el diagnóstico definitivo de superdotación tras un completo estudio multidisciplinar, y la segunda se refiere a la flexibilidad a la hora de establecer el diagnóstico definitivo.

\section{Fases del proceso de identificación}

Un gran número de expertos en superdotación (Clark, 1992; Feldhusen y Jarwarn, 1993; Davis y Rim, 1994; Treffinger y Feldhusen, 1996) admiten en el proceso de identificación dos fases:

a) screening, $\mathrm{y}$

b) diagnóstico.

Para estos autores el objetivo de la primera fase es determinar, en un principio, que sujetos son potencialmente capaces para integrarlos en un programa educativo concreto. Una vez determinado el posible grupo de sujetos, se pasaría al proceso de diagnóstico con la finalidad de seleccionar con mayor precisión quiénes se beneficiarán del programa para el que se está llevando a cabo la identificación. Para la elaboración de un diagnóstico es necesario un proceso amplio de medidas individuales de las características más relevantes de estos sujetos (Tourón y otros, 1998). Podríamos decir, pues, que la identificación va encaminada al total de alumnos (al grupo) y se realiza a través de pruebas generales y colectivas, mientras que el diagnóstico, que puede también comprender esa primera fase, tiene un carácter individual.

En la misma línea, Genovard y Castelló (1990) señalan también dos modalidades en el proceso de identificación de la superdotación:

1) Determinar el número de superdotados de un grupo de sujetos concretos (enfoque diferencial), lo que significa que el planteamiento se hace de forma grupal y los instrumentos utilizados son de uso general, $y$

2) Medir las capacidades y destrezas de los sujetos, modalidad que está orientada hacia el perfil del superdotado (enfoque específico).

Quizá convenga aclarar que no todos los alumnos que acuden a un diagnóstico individual pasan por una fase de selección, ya que son fácilmente identificables, tanto en el contexto escolar como en el familiar. Además, en España la primera fase mencionada (screening) se está desarrollando muy lentamente, por lo que la modalidad más frecuente es el diagnóstico individual. 


\section{La evaluación como elemento esencial para el diagnóstico}

La evaluación constituye uno de los momentos más importantes en la realización de un diagnóstico para poder realizar una correcta intervención educativa. Una evaluación inapropiada, que propicie un diagnóstico desajustado, en el que se detecte un superdotado cuando no lo es o viceversa, produciría graves problemas al no situar al alumno en el contexto de intervención más adecuado (Álvarez y Soler, 1996).

En el actual sistema educativo español son los profesionales de la Pedagogía y la Psicología, integrados en los Equipos de Orientación Educativa y Psicopedagógica y los Departamentos de Orientación de los centros docentes, quienes tienen la competencia para diagnosticar y determinar de manera formal si un alumno tiene necesidades educativas especiales asociadas a condiciones personales de superdotación intelectual, tal como recoge el Capítulo I de la Orden de 14 de febrero de 1996. Según este capítulo la información que debe reunir la evaluación psicopedagógica para identificar a un alumno superdotado es la siguiente (MEC, 2000:63):

\section{DATOS PERSONALES}

- Condiciones personales de sobredotación

- Competencia curricular

- Posibles desequilibrios (disincronías internas o sociales) entre lo intelectual, afectivo, psicomotor, lenguaje, relación con el entorno, etc.

- Autoconcepto

- Estilo de aprendizaje, concretando áreas, contenidos y actividades

- Habilidad para plantear y resolver problemas

- Tipo de metas que persigue

- Perseverancia en la tarea

- Ritmo de aprendizaje

- Historia educativa y escolar

\section{DATOS SOBRE EL CONTEXTO ESCOLAR}

- Análisis de las características de la intervención educativa

- Relaciones que establece con los profesores y con el grupo

- Organización de la respuesta educativa

3. DATOS SOBRE EL CONTEXTO FAMILIAR

- Características de la familia y de su entorno

- Expectativas de los padres

- Posibilidades de cooperación con la familia

- Recursos culturales y sociales de la zona

En la línea de la evaluación psicopedagógica, Hagen (1989) aconseja tener en cuenta algunas orientaciones que pueden ayudar en el diagnóstico de alumnos superdotados, como la concreción de los campos en los que se quiere valorar la superdotación, así como sus indicadores, determinar las fuentes de información y los instrumentos de medición para cada 
uno de los indicadores propuestos y fijar los criterios de interpretación de datos e informaciones conseguidas. Es decir, el diagnóstico sería la evaluación psicopedagógica y multidisciplinar que proporcione información relevante para la toma de decisiones sobre las medidas de intervención más adecuadas para dar respuesta a las necesidades del alumnado con capacidades excepcionales.

\section{El proceso diagnóstico en el tratamiento de la superdotación}

En la literatura especializada y desde enfoques diferentes la preocupación por el estudio del proceso diagnóstico en educación está presente en diversos autores (Anaya, 2002; Buisán y Marín, 1987; Granados, 2001, entre otros), proponiendo de una u otra forma la necesidad de seguir unas fases o etapas más o menos lineales para conseguir los fines propuestos con anterioridad acerca del objeto de estudio (Peña del Agua, 2003). Martínez González (1993:147) define el proceso diagnóstico como un método de investigación que, a través de un plan pensado y ordenado en etapas sucesivas, pretende llegar al conocimiento científico de una situación - entendiendo por ésta un sujeto, un grupo de sujetos, un ambiente, una metodología o una institución - con el fin de actuar sobre la misma. No obstante, cada proceso de diagnóstico puede presentar características diferenciadas en función de aspectos como: el modelo teórico al que se adscriba el profesional que lo realiza, las variables que elija para el estudio, el hecho de que el diagnóstico se centre en un sujeto, grupo, programa e institución (Padilla, 2002).

\section{Pasos del proceso diagnóstico}

En el caso del tema que nos ocupa, podemos establecer las fases o pasos del proceso diagnóstico de la siguiente manera (Peña del Agua, 2003):

1. Determinación previamente clara y específica de lo que se quiere medir (concepto de superdotación aceptado por la comunidad científica).

2. Planificación del procedimiento que se seguirá (selección de indicadores de superdotación y establecimiento de criterios para valorar las diversas informaciones logradas).

3. Recogida de información (exploración y comprobación).

4. Corrección e interpretación de los datos (descripción y explicación de los resultados)

5. Toma de decisiones (orientación/intervención)

6. Evaluación del seguimiento de las decisiones tomadas.

No obstante, la calidad del diagnóstico dependerá del rigor con que se haya realizado el proceso. Ese rigor será tanto mayor cuanto más se acerque el proceder diagnóstico al de la investigación científica. Tal proceder puede servir de marco referencial al que hay que acercarse lo más posible, pero en modo alguno pueden coincidir plenamente con el diagnóstico en casos reales, dado que la praxis diagnóstica conlleva una serie de actuaciones, puntos de mira y finalidades en los que la ciencia, puesta al servicio de las personas, debe sacrificarse en pro del beneficio y bienestar de los diagnosticados (Anaya, 2000: 81). 


\section{Técnicas e instrumentos de evaluación y diagnóstico}

Una cuestión muy importante relacionada con la identificación y el diagnóstico o evaluación psicopedagógica de los superdotados se refiere a la elección de aquellos instrumentos más adecuados que permitan recoger información relevante para identificar las habilidades y aptitudes que poseen los alumnos más capaces y los criterios a partir de los cuales se toman las decisiones necesarias y se realiza el diagnóstico correspondiente, con el fin de que educadores, orientadores y padres puedan planificar una mejor educación para estos niño (Sobrado, 1997).

\section{Importancia de la selección de instrumentos de medida para la evaluación diagnóstica}

Los procedimientos para la evaluación diagnóstica del superdotado no pueden limitarse a tests de inteligencia. Son muchos los autores (Coriat, 1990; Genovard y Castelló, 1990; Sobrado, 1997; Howell y otros, 1997) que, desde diferentes posiciones teóricas, consideran necesario utilizar un conjunto de técnicas, procedimientos e instrumentos que tengan en cuenta otros aspectos, además de experiencias vitales y educativas de esos sujetos. Las técnicas e instrumentos que se utilicen (Maganto, 1996) estarán en función de los objetivos de la evaluación, de la información proveniente de los instrumentos, de su validez y fiabilidad, de la edad de los sujetos, de la relación entre economía y tiempo, pero fundamentalmente del modelo teórico más o menos flexible con el que se trabaje.

En las prácticas con sujetos superdotadas (Genovard y Castelló, 1990) se usan instrumentos efectivos de evaluación como los inventarios, autoinformes, listas de control, datos biográficos, estudio de casos, observación, cuestionarios, escalas, entrevistas, etc., junto a los clásicos tests de inteligencia, aptitudes, rendimiento escolar y creatividad. En opinión de Sobrado (1997), entre las principales fuentes de información para la valoración de sujetos superdotados se pueden citar los tests en un sentido amplio, las listas de control, las nominaciones, la entrevista, la observación y evaluación de productos y las realizaciones. Para Howell y otros (1997) la práctica educativa actual impone un enfoque multifactorial de la evaluación que integra información proveniente de diversas fuentes: tests de inteligencia, evaluación de la creatividad, tests de ejecución, carpetas de trabajo y actividades, propuesta de los profesores, evaluación de los padres, propuesta de los compañeros y propuesta del propio alumno.

Respecto de los instrumentos susceptibles de ser utilizados para una exploración diagnóstica, Berché (1999) sugiere recurrir a los parámetros que han sido dictaminados por la Conferencia de Washington (1981). De forma general, el diagnóstico debe incluir al discente al menos en una de las cuatro categorías que a continuación exponemos:

1. Grupo de instrumentos denominados por la Conferencia de Washington como "no diagnóstico", que son aquellos que por sí solos no son suficientes para establecer un diagnóstico definitivo, sino que sólo se utilizan en fases de nominación.

2. Grupo de tests relacionados con los éxitos escolares. 
3. Tests de diagnóstico individual y medida exacta del nivel de inteligencia, que pueden ser usados para cualquier categoría de superdotación.

4. Cuestionarios de evaluación subjetiva y de observación.

Una vez analizadas estas y otras propuestas presentes en la literatura actual, pretendemos señalar, por un lado, algunos de los instrumentos de medida que recogen información cuantitativa y, por otro, diferentes fuentes de información de carácter cualitativo. La selección de los instrumentos de medida es un proceso amplio y complejo que requiere una gran atención, dedicación y conocimiento de los mismos.

\section{Instrumentos de medida (valoración cuantitativa)}

En este apartado mostramos algunos de los tests relativos a la medida de la inteligencia general, aptitudes específicas, creatividad y rendimiento académico, y que por razón de espacio únicamente enumeramos:

- Tests de inteligencia general: Las pruebas de inteligencia general aportan una medida operativa fiable y válida del rendimiento intelectual. Algunos de los principales tests de inteligencia empleados en los procesos de la evaluación diagnóstica son:

- Escala de Inteligencia Stanford-Binet (Terman, L. M. y Merrill, M. R.)

- Escala de Inteligencia Wechsler para Niños-Revisada (WISC-R) (Wechsler, D.)

- Test de Factor “G” (escalas 2 y 3) (Cattell, R. B. y Cattell, A. K.)

- Test de Matrices Progresivas de Raven (Raven)

- Aptitudes Cognoscitivas (Thornidike, Hagen y Lorge)

- Escala de Alexander (Prueba del Passalong)

- Sternberg Triarhic Abilities Tests (STAT)

- Tests de aptitudes específicas: Estas pruebas permiten conocer las aptitudes específicas de una persona y predecir el rendimiento en áreas concretas. A continuación enumeramos algunas de las mismas:

- Test de Aptitudes Diferenciales (DAT) (Bennett y otros) (cuatro de los ocho valores son fundamentales para valorar la superdotación: pensamiento verbal y abstracto, capacidad numérica y espacial).

- Tests de Aptitudes Mentales Primarias (PMA) (Thurstone y Thurstone)

- Escala de Aptitudes Mentales Primarias (AMPE-F) (F. Secadas)

- Test de Aptitudes Musicales (Seashore)

- Test de Aptitudes Artísticas (Meyer)

- Test de Artes Visuales (Lewerenz)

- Tests de creatividad: Tales pruebas valoran la flexibilidad para adaptarse a nuevos planteamientos, la divergencia de pensamiento y la búsqueda de soluciones ante nuevas propuestas. Entre los tests relacionados con la capacidad creativa de las personas, se encuentran los siguientes: 
- Test of Creative Thinking (Torrance)

- Test de Pensamiento Divergente (Guilford)

- Thinking Creativel with Sounds and Words (Creatividad Artística)

- Test of Creative Pontencial (Hoepfner y Hemenway)

- Thinking Creatively with Sounds and Words (Torrance, Khatena y Cunnington)

- Creativy and Intelligence: Exploration with Gifted Students (Getzels y Jackson)

- Test de Abreacción para Evaluar la Creatividad (TAEC) (S. de la Torre, 1991)

- Tests de rendimiento académico: Estos tests aprecian la medida en que una persona ha ejecutado algo, ha adquirido alguna información o ha realizado una tares. Citamos algunos de los mismos:

- Test de Habilidades Básicas (IOWA)

- Test de Aptitudes Cognitivas Superiores (Ross)

- Batería de Aptitudes para el Aprendizaje Escolar (BAPAE)

- Reasoning Test (SAT I) (Razonamiento verbal y matemático) y (SAT II) (Se ocupa de diferentes áreas de conocimiento)

- Valoración del Pensamiento Crítico (Watson-Glasser)

- Cuestionario de Estrategias de Aprendizaje (CUES)

Estos y otros tests que se utilizan en la evaluación psicopedagógica pueden presentar dificultades que el profesional ha de conocer y tener en cuenta tanto en el momento de la selección como de la aplicación de los mismos.

\section{Criterios en la selección de los tests}

Los instrumentos de medición - tests- pueden presentar problemas tanto en lo concerniente a su configuración como en la fiabilidad de su medida. Feldhusen y Jarwan (1993) han señalado una serie de criterios que se deben tener en cuenta a la hora de seleccionar estos instrumentos de evaluación diagnóstica y que nosotros describimos de manera concisa:

- Relevancia del test: Hace referencia a la relación que debe darse entre lo que se pretende medir y los contenidos de la prueba.

- Baremo: Proporcionan un marco de referencia mediante el cual adquieren significado las puntuaciones obtenidas en un test.

- Fiabilidad: Indica el grado de precisión de la medida.

- Validez: Se refiere al grado de exactitud con que un test mide efectivamente la característica que se propone medir.

- Efecto techo: Límite superior de la aptitud que se puede medir con un test, es decir, que pueda discriminar la diferencia entre los sujetos a partir de un determinado nivel.

Entre los inconvenientes de las medidas estandarizadas utilizadas generalmente para el diagnóstico formal suelen darse limitaciones por la parte superior del instrumento o, dicho con otras palabras, tienen un "techo bajo", lo que significa que la puntuación máxima del instrumento es insuficiente para la competencia de los sujetos excepcionales. Por eso no 
suelen proporcionar una medición precisa de las variables de dichos sujetos, sino que solamente permiten discriminar entre estos sujetos y los normales intelectualmente (Feldhusen, 1985). Las medidas específicas son una solución a este problema, ya que están construidas para una población excepcional y por tanto están en función de sus parámetros propios, por lo que el techo es superior al de los instrumentos de uso general; también el tipo de variables medidas es generalmente diferente. Desde una perspectiva multidimensional de la superdotación es necesaria una combinación adecuada, tanto de instrumentos de medida como de otras fuentes de información, por lo que a continuación presentamos las fuentes de información más relevantes:

\section{Fuentes de información (valoración cualitativa)}

En este apartado se exponen las fuentes de información más utilizadas por los profesionales del diagnóstico:

- Información escolar: La denominación por parte de los profesores es muy importante, éstos colaboran en gran medida a completar el diagnóstico, al ser quienes mejor conocen a los alumnos y quienes los derivan a los especialistas que integran los Departamentos de Orientación con la finalidad de que sean diagnosticados. No obstante, puede suceder que los juicios emitidos por los profesores estén sesgados debido a criterios de rendimiento académico o por carencias en la formación sobre el tema de la superdotación intelectual. Richert (1991) señala al respecto que los datos procedentes de esta fuente han de contemplarse como una información complementaria de los datos obtenidos en los tests, y no tanto como una información confirmatoria de los mismos.

- Información familiar: Los padres son una valiosa fuente de información puesto que son los primeros en darse cuenta de ciertas conductas poco comunes en sus hijos en comparación con otros de su misma edad. Son una fuente de información bastante fiable, especialmente de aspectos evolutivos y aptitudinales, puesto que su situación les permite observar conductas que no aparecen en otros contextos (Genovard y Castelló, 1990). Por otro lado, puede suceder que los juicios de los padres tiendan a ser un tanto desmesurados, o por el contrario, que no aprecien las altas capacidades de sus hijos por desconocimiento (Davis y Rimm, 1994).

- Información social: Los compañeros de la clase constituyen una fuente de información relevante, ya que entre los "iguales" es donde manifiestan conductas o rasgos de liderazgo. La nominación entre compañeros tiene gran importancia, sobre todo para la valoración de la creatividad en función de las realizaciones en la clase, respuestas y soluciones a problemas, juegos, etc. Los "pares" poseen unos buenos fundamentos para apreciar la originalidad, excepcionalidad e imaginación de sus compañeros (Sobrado, 1997). También aquí conviene advertir la importancia de la edad de los alumnos que van a participar en el criterio de selección de superdotados. El nivel evolutivo puede suponer una dificultad a la hora de diferenciar con claridad entre el compañero que mejor realiza una actividad, poseedor de altas capacidades, y aquel que es su mejor amigo y lo considera con cualidades extraordinarias como amigo suyo que es (Rayo, 2001). 
- Información personal: La importancia de implicar a los estudiantes en su propio diagnóstico ha sido señalada por Treffinger y Feldhusen (1996) para identificar sus puntos fuertes y débiles, sus talentos y sus intereses.

Para Genovard y Castelló (1990) el criterio de diferenciación desde el enfoque cuantitativo viene dado por "menor que" o "mayor que", de forma que las diferencias entre los valores de la distribución serán exclusivamente explicables por la variable descrita. Por el contrario, la perspectiva cualitativa implica una situación distinta, las variables cualitativas manifiestan unas propiedades de diferenciación objetiva, basada en la naturaleza de la propia variable, complementadas por la frecuencia de aparición de cada una de ellas. Para los citados autores, en el contexto cuantitativo el término "excepcional" no implica directamente diferencia, sino gradación, por lo que se pueden constatar las mismas propiedades en todos los valores, aunque con un grado de manifestación distinto.

\section{Procedimientos para la integración de la información}

Existe abundante literatura de cómo se debe llevar a cabo los procesos de identificación de carácter multidimensional desde un concepto amplio de superdotación como medida para proporcionar ayudas muy diversas al mayor número posible de sujetos. Sin embargo, apenas disponemos de estudios relativos a cómo se debe sintetizar la información, a pesar de la especial importancia la cuestión de cómo unificar todos los datos obtenidos en una puntuación global que ayude a tomar la mejor decisión posible (Rayo, 2001). Uno de los sistemas utilizados para integrar la información con operatividad y rigor y, en consecuencia, para decidir sobre el programa más adecuado, es el método de estudio de casos (Feldusen y Jarwan, 1993).

\section{El estudio de casos como estrategia de investigación}

El estudio de casos se le reconoce como una estrategia de investigación ideográfica encaminada a la toma de decisiones, que centra su atención en la indagación de un caso y que requiere un examen detallado de una situación, de un único sujeto o de un evento particular. Afronta la realidad mediante un análisis detallado de sus elementos y la interacción que se produce en ellos y su contexto, para llegar, mediante un proceso de síntesis, a la búsqueda de significado y a la toma de decisiones. Ary y otros (1987), señalan posibles objetivos del estudio de casos, entre los que destacamos los siguientes:

a) Describir y analizar situaciones únicas, como puede ser el caso de un niño superdotado.

b) Diagnosticar una situación, para orientar, llevar a cabo un asesoramiento, etc.

c) Completar la información aportada por investigaciones estrictamente cuantitativas.

Para los citados autores el potencial del estudio de casos radica en que permite centrarse en un caso o situación concreto e identificar los distintos procesos interactivos que lo conforman. Bartolomé (1983), recoge aspectos fundamentales presentes en el estudio de casos: 
- Amplitud de los datos.

- Datos de diferentes niveles (sociales, psicológicos, educativos).

- Formación de índices y tipos en los que los diversos aspectos estudiados se estructuran formando una unidad.

- Interacción dentro de una dimensión de tiempo: se observan los procesos y cambios que sufren las variables en el tiempo.

El estudio de casos analiza la información a través de la valoración de expertos y normalmente no incluye una cuantificación numérica (Maker, 1989). En el método de estudio de casos se deben evitar los juicios excesivamente cualitativos o poco fundados. No parece que la valoración cualitativa se deba oponer a la información cuantitativa obtenida por instrumentos adecuados, pero se considera de gran importancia que la acción de los expertos en este método esté lo más fundada posible en informaciones fiables sobre los sujetos. Ahora bien, hay que tener en cuenta las limitaciones metodológicas que presenta el estudio de casos, ya que se plantean dificultades respecto a la generalización y falta de rigor científico que influyen en los resultados y conclusiones (Maganto, 1996).

\section{Ventajas e inconvenientes del estudio de casos}

De las aportaciones realizadas sobre el estudio de casos por Feldhusen y Jarwan (1993) se deduce que el principal postulado subyacente a este procedimiento es que un índice global de superdotación es mejor que la suma mecánica de puntuaciones individuales en diferentes medidas de identificación. Este método, de carácter holístico, implica que el todo es distinto de la suma de las partes y analiza la información a través de las valoraciones de expertos; valoración que emerge al analizar el conjunto de la información disponible, y de ahí se desprenden sus ventajas y los inconvenientes. En todo caso se realiza un juicio global sobre cada sujeto, no guiado únicamente por los datos sino teniendo en cuenta un marco de referencia más amplio (Jiménez Fernández, 2001).

Kratochwill (1978), hace alusión a las dificultades del método de estudio de casos y las resume en las siguientes:

a) falta de atención a la validez, tanto interna como externa;

b) limitadas opciones de diseño; y

c) dificultad para generalizar los hallazgos.

En el estudio de casos se da cierta sistematización que sin obligar al evaluador a avanzar hipótesis provisionales tras el análisis de la información disponible, pretende seleccionar las más verosímiles para tener éxito en el programa educativo.

Con todos los inconvenientes que se puedan dar en el de estudio de casos, este método es más fiable que confiar a un solo criterio o instrumento la decisión final, particularmente si el estudio de casos es efectuado por un grupo de expertos (Tourón y otros, 1998). Según los citados autores, este método es recomendable siempre que los profesionales que lo utilicen tengan una adecuada formación, y que sus acciones estén lo más fundadas posible en informaciones fiables sobre los sujetos objeto de diagnóstico. 


\section{A modo de síntesis}

En este apartado sintetizamos algunos de los aspectos expuestos a lo largo del presente artículo y que nos parecen especialmente importantes:

1. La superdotación debe ser entendida dentro del marco de atención a la diversidad, vista desde la perspectiva de educar a los estudiantes en la diversidad, orientada al desarrollo progresivo de sus estrategias cognitivas y metacognitivas, pero haciendo hincapié en las características personales.

2. La superdotación intelectual no es un atributo unidimensional, sino un fenómeno multidimensional que se manifiesta de distintas formas y en diferentes niveles según las personas, las circunstancias y el momento de que se trate. No es por lo tanto algo estático, sino que evoluciona a lo largo del desarrollo de un sujeto como resultado de la interacción entre la capacidad innata y el apoyo ambiental apropiado.

3. Es muy importante que el superdotado haya sido identificado, $y$, consecuentemente, diagnosticado por profesionales especializados que sepan discriminar los instrumentos de medida más adecuados y las fuentes de recogida de información más relevantes, así como la metodología de estudio para una mejor integración de la información para llegar a la toma de decisiones. Ello permitirá situar a los sujetos en los programas adecuados para que desarrollen de manera óptima sus aptitudes

Por último, señalamos con Freeman (1988) que el rendimiento excepcional de un sujeto no depende sólo de la dotación o capacidad innata superior, sino que está asociada con ciertas características de personalidad y exige unas condiciones que posibiliten el desarrollo y actualización de esta capacidad.

\section{Referencias bibliográficas}

Álvarez Pérez, L. y Soler, E. (1996). La diversidad en la práctica educativa. Modelos de acción tutorial, orientación y diversificación. Oviedo, Aula Abierta-Monografía 27.

Anaya, D. (2000). Diagnóstico en orientación e intervención psicopedagógica. Bases conceptuales y metodológicas. Madrid, UNED ( $1^{\mathrm{a}}$ reimp.).

Anaya, D. (2002). Diagnóstico en Educación. Madrid, Sanz y Torres.

Ary, D., Jacobs, L.C. y Razavieh, A. (1987). Introducción a la investigación pedagógica. México: Interamericana.

Bartolomé, M. (1983). Pedagogía Diferencial. Aproximación a una ciencia. Barcelona, Universidad de Barcelona.

Berché, J. (1999). La superdotación infantil. Del mito a la realidad. Barcelona: ISEP Universidad

Bloom, B. (1985). Developing talent in young people. New York: Ballantine Books.

Buisán, C. y Marín, M. A. (1987). Cómo realizar un diagnóstico pedagógico. Barcelona, Laertes.

Clark, B. (2000). "Optimizar el aprendizaje: Identificar, planificar y atender a los niños y jóvenes superdotados". En IDEACCIÓN, No Especial, (pp. 13-21). Valladolid, Centro "Huerta del Rey".

Clark, B. (1992). Growing up Gifted: Developing the Potential of Children at home and at School. Los Ángeles: California State University.

Coriat, A. R. (1990). Los niños superdotados. Barcelona, Herder. 
Davis, G. A. y Rimm, S. B. (1994). Education of the Gifted and Talented. Englewood Cliffs, N. J.: Prentice Hall, ( $3^{\mathrm{a}}$ edición).

Diamond, M. (1988). Enriching heredity: The impact $f$ the environment on the anatomy of the brain. New York: The Free Press.

Feldhusen, J. F. (1985). Toward Excellence in Gifted Education. Denber, CO: Love.

Feldhusen, J. F. y Jarwan, F. A (1993). Identification of Gifted and Talented Youth for Educational programs. En K. A. Hellen, F. J. Mönks y A. H. Passow (Eds.): International Handbook of Research and Development of Giftedness and Talent. Oxford: Pergamon Press.

Freeman, J. (1988). Los niños superdotados. Aspectos psicológicos y pedagógicos. Madrid, Santillana.

Granados, P. (2001). Diagnóstico pedagógico. Madrid, UNED (Cuarta reimpresión).

García Yagüe, J. y otros (1986). El niño bien dotado y sus problemas. Madrid, CEPE.

Genorvard, C. y Castelló, A. (1990). El límite superior. Aspectos psicopedagógicos de la excepcionalidad intelectual. Madrid, Pirámide.

Hagen, E. (1989). Identification of the Gifted. New York: Teachers College Press.

Hany, E. A. (1993). "Methodological problems and issues concerning identification". En K. A. Heller, F. J. Mönks y A. H. Pasow (Eds.): International handbook of research and development of giftedness and talent (pp.) Oxford: Pergamon.

Howell, R., Hewards, W, Swassins, R. (1997). "Los alumnos superdotados". En W. L. Heward: Niños excepcionales. Una introducción a la educación especial (cap. 12). Madrid, Hall (5a ed.).

Jiménez Fernández, C. (2001). Diagnóstico y educación de los más capaces. Madrid, UNED.

Kandel, E. y Schwartz, J. (1991). Principles of Neural Science. New York: New American Library.

Kratochwill, T. T. (1978). Single subject research. Strategies for evaluating change. New York: Academic Press.

Maganto, J. M. (1996). Diagnóstico en Educación. Servicio Editorial Universidad del País Vasco.

Maker, C. J. (1989). "Educación del superdotado: tendencias significativas". En R. J. Morris y B. Blatt: Educación Especial. Investigaciones y tendencias (pp. 130-159). Buenos Aires, Ed. Médica-Panamericana.

Martínez González, R. A. (1993). Diagnóstico Pedagógico. Servicio de Publicaciones de la Universidad de Oviedo.

MEC (2000). Alumnos precoces, superdotados y de altas capacidades. Madrid, Secretaría General Técnica. Centro de Publicaciones.

Orden Ministerial del 14 de febrero de 1996, de la Evaluación psicopedagógica y procesos de escolarización de los alumnos con NEE (BOE de 23/02/96).

Padilla, M. T. (2002). Técnicas e instrumentos para el diagnóstico y la evaluación educativa. Madrid, CCS.

Peña del Agua, A. M. (2003). Proyecto docente. Perfil de Diagnóstico y Evaluación en Educación. Oviedo: Facultad de Ciencias de la Educación. Universidad de Oviedo (documento no publicado).

Rayo, J. (2001). Quiénes y cómo son los superdotados. Implicaciones familiares y escolares. Madrid, EOS.

Sobrado, L. (1997). "Los superdotados: evaluación psicopedagógica”. En M. Álvarez y R. Bisquerra (Coords.): Manual de Orientación y Tutoría (pp. 258/1-13). Barcelona, Praxis.

Tourón, J., Peralta, F. y Repáraz, Ch. (1998). La superdotación intelectual: modelos, identificación y estrategias educativas. Pamplona, EUNSA. 
Treffinger, D. J. y Feldhusen, J. F. (1996). Talent Recognition and development: Successor to Gifted Education. Journal for the Education of the Gifted, 19 (2), 181-193.

Webb, J. (1993). "Nurturing Social-Emotional Development of Gifted Children". En K. A. Heller, F. J. Mönks y A. H. Passow (Eds.): International Handbook of Research and Development of Giftedness and Talent (pp. 525-538). Oxford, Pergamon.

Whitome, J. (1988). "Nuevos retos a los métodos de identificación habituales". En Freeman, J. (D.): Los niños superdotados. Aspectos psicológicos y pedagógicos (pp. 115-138). Madrid, Santillana.

Fecha de recepción: 21-12-04

Fecha de revisión: 15-09-05

Fecha de aceptación: 19-10-05 